\title{
Activation transport under quantum Hall regime in HgTe-based heterostructure
}

\author{
S.V. Gudina ${ }^{1}$, V.N. Neverov ${ }^{1}$, E.G. Novik ${ }^{2}$, E.V. Ilchenko ${ }^{1}$, G.I. Harus ${ }^{1}$, N.G. Shelushinina ${ }^{1}$, \\ S.M. Podgornykh ${ }^{1,3}$, M.V. Yakunin ${ }^{1,3}$, N.N. Mikhailov ${ }^{4,5}$, and S.A. Dvoretsky ${ }^{4,6}$ \\ ${ }^{1}$ M.N. Mikheev Institute of Metal Physics, Ural Branch, Russian Academy of Sciences \\ 18 S. Kovalevskaya Str., Ekaterinburg 620137, Russia \\ E-mail: ilchenko@imp.uran.ru \\ ${ }^{2}$ Physikalisches Institut (EP3), University of Würzburg, Würzburg 97074, Germany \\ ${ }^{3}$ B.N. Yeltsin Ural Federal University, 19 Mira Str., Ekaterinburg 620002, Russia \\ ${ }^{4}$ A.V. Rzhanov Institute of Semiconductor Physics, Siberian Branch, Russian Academy of Sciences \\ 13 Akad. Lavrent'eva Ave., Novosibirsk 630090, Russia \\ ${ }^{5}$ Novosibirsk State University, 2 Pirogova Str., Novosibirsk 630090 Russia \\ ${ }^{6}$ National Research Tomsk State University, 36 Lenina Ave., Tomsk 634050, Russia
}

Received December 6, 2016, published online February 24, 2017

\begin{abstract}
We have measured the temperature $(2.9 \mathrm{~K}<T<50 \mathrm{~K})$ and magnetic field $(0 \mathrm{~T}<B<9 \mathrm{~T})$ dependences of longitudinal and Hall resistivities for $\mathrm{HgCdTe} / \mathrm{HgTe} / \mathrm{HgCdTe}$ system with $\mathrm{HgTe}$ quantum well width of $20.3 \mathrm{~nm}$. The activation analysis of the experimental magnetoresistivity traces has been used as a quantitative tool to probe inter-Landau level distances. The activation energies were determined from the temperature dependence of the longitudinal resistivity in the regions of quantized Hall plateaus (for the filling factors $v$ of 1,2 and 3) and the indications of the large values of the $g$ factor $\cong 30-75$ were found.

PACS: 73.63.Hs Quantum wells;

73.43.Qt Magnetoresistance.
\end{abstract}

Keywords: quantum well, activation magnetotransport, effective mass, $g$ factor.

\section{Introduction}

Heterostructures of $\mathrm{HgTe}$ and $\mathrm{CdTe}$ are of type III combining a positive band gap semiconductor ( $\mathrm{CdTe})$ and a zero-gap semimetal ( $\mathrm{HgTe})$ with gaps formed between states of $\Gamma 8$ and $\Gamma 6$ symmetry. In recent years, high-mobility HgTe-based heterostructures have become available for experimental investigations due to advances in the molecular beam epitaxy (MBE) technology (see, for example, [1] and references therein). Layered quantum structures of $\mathrm{HgTe}$ and CdTe attracted interest due to the rich possibilities for band structure species.

Depending on their thicknesses, single $\mathrm{HgTe}$ quantum well (QW) between CdTe (or $\mathrm{HgCdTe}$ with a large energy gap) barriers can exist in two distinct regimes [1]. For small QW widths and thus larger subband separations, an open gap is formed between heavy-hole subband $(H 1)$ and an electron-like light-particle subband $(E 1)$ that corresponds to the normal regime. With increasing QW width the $E 1$ subband falls below the topmost heavy-hole subbands and takes on hole-like character, while the $H 1$ subband becomes electron-like. Such QWs are in the inverted-band regime. The inverted regime is achieved when $\mathrm{QW}$ width, $d_{Q W}$, exceeds a critical value $d_{c} \cong 6.3 \mathrm{~nm}$.

In the latter case the conduction band exhibits $\Gamma 8$ symmetry with a coupling heavy-hole to light-particle states at finite inplane wave vectors. That leads to a complex Landau level (LL) spectra as a quantizing magnetic field uncovers the mixed nature of the $H 1$ subband in the invertedband regime [2].

It is known that the effective probing of the LL fan diagram can be realized by means of the activation mag- 
netotransport analysis under the quantum Hall $(\mathrm{QH})$ regime. Distances between adjacent LLs can be extracted and a density of localized states in the mobility gaps may be determined from scanning them by the Fermi level in experiments of this kind [3-7]. Experimental reconstruction of the energy spectrum is especially important for systems with a complex band structure where the LL picture is determined not only by the cyclotron energy with a given effective mass as for a simple parabolic conduction band system.

As for the HgTe-based 2D structures, in [8] the activation energies were determined from the temperature dependence of the longitudinal resistivity in the regions of quantized Hall plateaus (for the filling factors $v$ of 1 and 2) in a $6.6 \mathrm{~nm} \mathrm{HgTe}$ quantum well at magnetic fields up to $34 \mathrm{~T}$ at nitrogen temperatures. The indications of the large values of the $g$ factor (about 30-40) were found.

In [9] the quantum Hall effect (QHE) in HgTe QWs with a finite band gap below and above the critical thickness $d_{c}\left(d_{Q W}=5.9 \mathrm{~nm}\right.$ and $\left.11 \mathrm{~nm}\right)$ has been studied up to temperatures of the order of $50 \mathrm{~K}$. From temperature dependent magnetotransport measurements, they extracted the energy gaps between LLs of the order of $40-45 \mathrm{meV}$ for $v=1$ and of the order of $25 \mathrm{meV}$ for $v=2$, in good agreement with the Landau level spectrum obtained from $\mathbf{k} \cdot \mathbf{p}$ calculations.

In our previous paper [10] we have studied the QHE plateau-plateau transition for a wide temperature range $T=2.9-50 \mathrm{~K}$ at $\mathrm{HgCdTe} / \mathrm{HgTe} / \mathrm{HgCdTe}$ system with the inverted band structure. In subsequent work [11] the first study of variable-range hopping conductivity at the QHE plateau regions in the $\mathrm{HgTe} / \mathrm{HgCdTe}$ heterostructure has been done. The present work is devoted to a probing of HqTe quantum well LL spectrum at the same system by means of the quantum Hall effect.

\section{Characteristics of the sample}

We present a study of quantum magnetotransport in a 20.3-nm-wide HgTe QW grown on the (013) GaAs substrate, symmetrically modulation doped with In at both sides at distances of about $10-\mathrm{nm}$ spacers. The electron gas density is $n_{S}=1.5 \cdot 10^{11} \mathrm{~cm}^{-2}$ with a mobility of $22 \mathrm{~m}^{2} /(\mathrm{V} \cdot \mathrm{s})$.

The 2DEG in wide HgTe quantum well $\left(d>d_{c} \cong\right.$ $\cong 6.3 \mathrm{~nm}$ ) is characterized by a highly specific energy spectrum with an inverted band structure, low effective mass $m_{\text {eff }} \approx(0.02-0.03) m_{0}$ ( $m_{0}$ being free electron mass) and, consequently, a large Landau level separation. In [12] a review of the bulk HgTe and CdTe bandstructure and a simple model for the physics of the relevant subbands of $\mathrm{HgTe} / \mathrm{CdTe} \mathrm{QW}$ are presented.

In the inverted regime of $\mathrm{HgTe} \mathrm{QW}$ the first sizequantized heavy-hole subband $H 1$ becomes the lowest conduction band as the theory $[13,14]$ predicts an electronlike effective mass for it. The highest valence band is now the second size-quantized heavy-hole subband $H 2$ with nonmonotonic dispersion law [13,14].

A substantial overlap about $6.45 \mathrm{meV}$ of the valence $H 2$ and conduction $H 1$ subbands is obtained when the strain is considered in calculations, but this overlap would not be felt experimentally in a single QW at electron densities $n \gtrsim 1.5 \cdot 10^{11} \mathrm{~cm}^{-2}$ since the Fermi level is above the overlap region.

In the system we studied the conduction is carried out just by the electrons of band $H 1$ with extremely small value of the effective mass of carriers: for $n_{S}=1.5 \cdot 10^{11} \mathrm{~cm}^{-2}$ mass at the Fermi level $m_{\text {eff }}=(0.022-0.026) m_{0}[15,16]$ and with a large value of $g$ factor, $g \cong 50$ [16].

The Landau level spectra for investigated structure $\mathrm{HgTe} / \mathrm{Hg}_{0.35} \mathrm{Cd}_{0.65} \mathrm{Te}([001])$ QW calculated within the axial approximation is shown on Fig. 1. The LL notations correspond to the notations of [2].

It is seen that the Landau levels are of the essence no equidistant and nonlinear on magnetic field which caused by the mixed nature of the $H 1$ and $H 2$ subbands in the inverted-band regime due to a coupling between heavy-hole to light-particle states at finite inplane wave vectors $k_{\|}$. Only the lowest Landau level $(N=-2 \downarrow)$ of the $H 1$ subband contains pure heavy-hole states which do not mix with the light-particle states [9] (see also [17] and references therein).

In [17] it is shown that this level is of the same nature in two and in three dimensions and it is mapped on the $b$ set level of Guldner et al. [18] $E_{b}(0)=(e \hbar B / m) \varepsilon_{b}(0)$, where

$$
\varepsilon_{b}(0)=\frac{1}{2}\left(\gamma_{1}+\bar{\gamma}\right)-\frac{3}{2} \kappa
$$

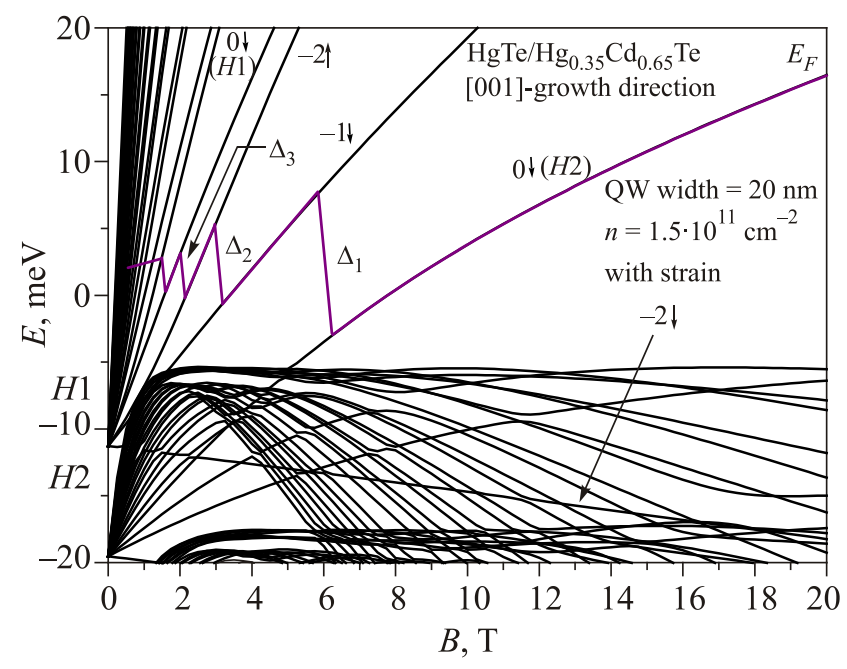

Fig. 1. (Color online) Landau levels of $H 1$ and $H 2$ subbands for an $n$-type $\mathrm{HgTe} / \mathrm{Hg}_{0.3} \mathrm{Cd}_{0.7} \mathrm{Te}(001) \mathrm{QW}$ as a function of magnetic field within the axial approximation; $d_{W}=20 \mathrm{~nm}, n_{2 D E G}=$ $=1.5 \cdot 10^{11} \mathrm{~cm}^{-2}$. The Landau levels are labeled in accordance with the notations of [2]: the quantum numbers $N=-2,-1,0, \ldots$, and the arrows $(\uparrow, \downarrow)$ indicate the dominant spin orientation of the state. The thick line represents the chemical potential. 
For the set of $\Gamma_{8}$ Luttinger parameters for $\mathrm{HgTe}$ $\left(\gamma_{1}=12.8, \bar{\gamma}=8.4\right.$ and $\left.\kappa=10.5[18]\right)$ we have

$$
3 \kappa>\left(\gamma_{1}+\bar{\gamma}\right),
$$

and, according to (1), the level $N=-2 \downarrow$ lowers its energy linearly with increasing magnetic field revealing a holelike character. All the other Landau levels of the $H 1$ subband show an electron-like character: they rise in energy with magnetic field due to the coupling with light-particle states.

Figure 1 shows also that the Landau level of the $H 2$ subband with $N=0 \downarrow$ becomes the highest $H 2 \mathrm{LL}$ at $B \gtrsim 5 \mathrm{~T}$ in consequence of a distinct mixing of the heavy and the light states [19]. The unusual behavior of $N=-2 \downarrow$ level from the conduction subband $H 1$ in inverted band HgTe QWs together with the peculiar dispersion of the $N=0 \downarrow$ level from the topmost valence subband $H 2$ leads to a crossing of conduction- and valence-subband states at a some value $B_{c}$ of the magnetic field (see Fig. 1).

Such behavior is specific for HgTe QWs and has been examined theoretically and experimentally (see, for example, [20]). In our structure the lowest $N=-2 \downarrow$ LL of the $H 1$ conduction subband and the $N=0 \downarrow$ LL of the $H 2$ valence subband cross at $B_{c} \approx 2.5 \mathrm{~T}$, and the point of intersection is immersed in the thick of valence band Landau levels (see Fig. 1). As a consequence, the $N=-2 \downarrow$ level is irrelevant in our situation and in the actual region of our study at $B>>B_{C}$ just the $N=0 \downarrow$ level of the $H 2$ subband appears as the lowest Landau level of the conduction band.

Thus, a peculiarity of HgTe QWs in the inverted-band regime is that nominally (in labeling of the Landau level by the dominant spin component at energies near the $H 1$ bottom) the spin splitting in the $H 1$ conduction subband turns out to be larger than the orbital splitting which is particularly evident from a scheme on the inset of Fig. 4. Such a relation of spin and cyclotron splittings is a consequence of specific numerical values for Luttinger parameters in $\mathrm{HgTe}$ (see Eqs. (1), (2)).

Note, that for $\mathrm{Ge}$ the values of $\Gamma_{8}$ Luttinger parameters $\left(\gamma_{1}=13.38, \bar{\gamma}=4.96\right.$ and $\left.\kappa=3.41[21]\right)$ are such that

$$
3 \kappa<\left(\gamma_{1}+\bar{\gamma}\right)
$$

and thus for the QW based on $p$-Ge the highest valence Landau level $E_{b}(0)=-(e \hbar B / m) \varepsilon_{b}(0)$ have the same energy dispersion as all other hole LL of a spatially quantized valence band [17].

Our further plan is as follows: first, to determine the activation energies of longitudinal resistivity $\rho_{x x}$ in the QHE regime for $v=1$ (the gap between $N=0 \downarrow$ LL of $H 2$ subband and $N=-1 \downarrow$ LL of the $H 1$ subband), $v=2$ (gap $N=-1 \downarrow \rightarrow N=-2 \uparrow$ of the $H 1$ subband) and $v=3$ (gap $N=-2 \uparrow \rightarrow N=0 \downarrow$ of the $H 1$ subband) and then, to estimate the background density of states (DOS) in these mobility gaps.

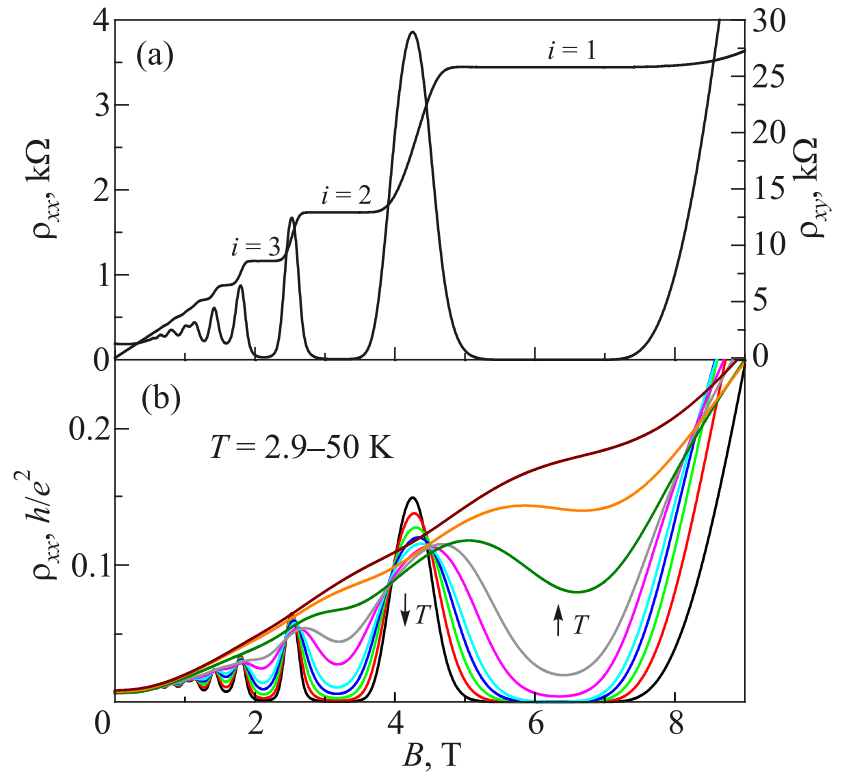

Fig. 2. (Color online) The longitudinal and Hall resistivity dependencies on the magnetic field $B$ at $2.9 \mathrm{~K}$ ( $i$ signifies a number of the QHE plateau) (a). The longitudinal resistivity at $T=2.9-50 \mathrm{~K}(\mathrm{~b})$.

\section{Experimental results and discussions}

We used the method of thermally activated magnetoresistivity for reconstruction of the 2D electron gas (2DEG) spectrum under quantizing magnetic fields at a wide $\mathrm{HgTe} \mathrm{QW}$ with the inverted band structure at $\mathrm{HgCdTe} / \mathrm{HgTe} / \mathrm{HgCdTe}$ system. Measurements of the longitudinal $\rho_{x x}$ and Hall $\rho_{x y}$ resistivities have been carried out in magnetic fields up to $9 \mathrm{~T}$ at $T=2.9-50 \mathrm{~K}$ (Fig. 2).

Figure 2(a) gives the magnetic-field dependences of the $\rho_{x x}$ and $\rho_{x y}$ resistivities for the sample under study at $T=2.9 \mathrm{~K}$. We can see the features characteristic of the QHE regime, i.e., the regions of plateau in the $\rho_{x y}(B)$ dependences with corresponding deep minimum of $\rho_{x x}(B)$ for filling factors up to $v=3$.

Figure 2(b) shows the magnetic-field dependences of the longitudinal $\rho_{x x}$ resistivity for investigated sample at $T=2.9-50 \mathrm{~K}$. The thermally activated conduction may be seen in the magnetic field range of the Hall plateaux.

\subsection{Activation energies}

The appearance of quantized plateaux in the $\rho_{x y}(B)$ dependences with vanishing values of $\rho_{x x}$ is now commonly accepted to be caused by the existence of disorder-induced mobility gaps in the DOS of a 2D system in high magnetic field.

When the Fermi level in the QHE regime is settled down in the gap between adjacent LLs, the thermally activated behavior of $\rho_{x x}$ (or $\sigma_{x x}$ ) is observed due to the excitation of electrons to the narrow band of extended states (with a width $\Gamma$ ) near the middle of a disorder-broadened LL. 
As a rule, an assumption is used that the delocalized states with discrete energies $E=E_{N}$ are separated by the (mobility) gap $\Delta \gg k_{B} T$, that leads to an expression [3-5]:

$$
\sigma_{x x}(T) \sim \exp \frac{-E_{A}}{k_{B} T}
$$

with $E_{A}=\left|E_{F}-E_{N}\right|$.

Activation behavior of the conductivity $\sigma_{x x}(T)$ for investigated sample in vicinity of $v=1$ and 2 is illustrated by Figs. 3(a), (b). Figures show a fit of $\sigma_{x x}(T)$ dependencies by the Arrhenius equation (4) (straight lines in figure) in the range of more than one (for $v=2$ ) or even three (for $v=1$ ) orders of conductivity at $T>10 \mathrm{~K}$. Deviations of experimental points from straight lines for $T \leq 10 \mathrm{~K}$ are explained by variable range hopping among localized states at $E_{F}$, which usually dominates for sufficiently low $T$ (see Ref. 11).

The activation energy $E_{A}$ as a function of $B$ for the filling factor $v$ in the vicinity of $v=1, v=2$ and $v=3$ for investigated sample is presented in Fig. 4. The activation energy achieves its maximum value $E_{a}^{\max }$ at integer value of $v$. The mobility gap width estimated as $\Delta=2 E_{a}^{\max }$ is closely related to the energy separation between the adjacent LLs: $\Delta=\left|E_{N}-E_{N^{\prime}}\right|$ within the uncertainty of the order of $\Gamma$. A scheme of the orbital and spin splitting for the $H 1$ conduction subband is given at the inset.

Figure 4 shows a comparison of the extracted activation energies with theoretical calculations of the LL dispersions from the Fig. 1 the calculated values of halfgaps, $\Delta_{v} / 2$, for $v=1,2$ and 3 are marked by asterisks on the figure (see also Table 1). It is seen that the experimentally and theoretically obtained energy gaps are in reasonably good agreement and thus the behavior of the sample is well described

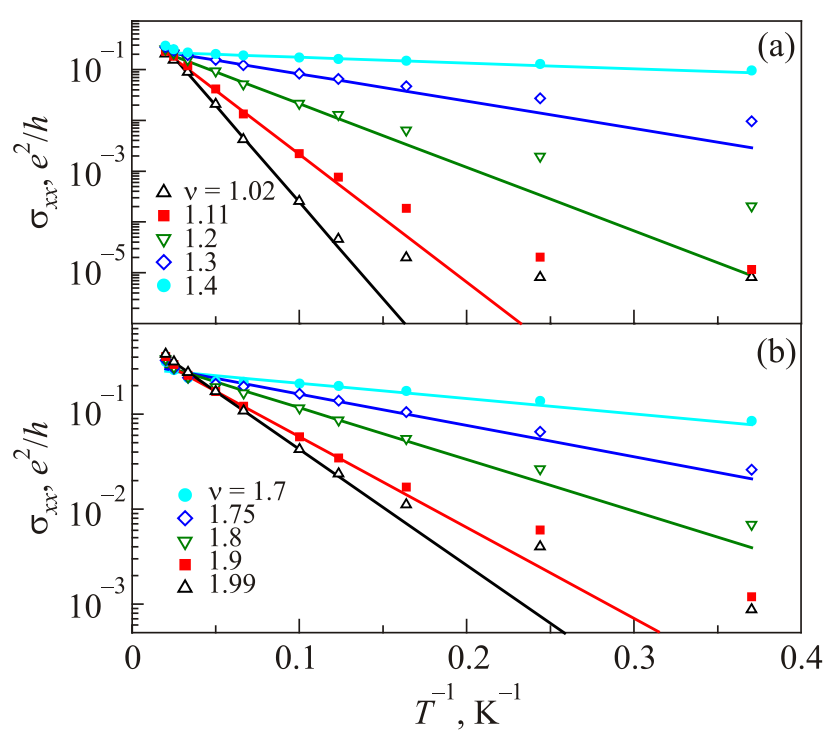

Fig. 3. (Color online) Plot of $\sigma_{x x}$ versus $1 / T$ in semi logarithmic scale at the high (a) and the low (b) field side of $1 \rightarrow 2$ transition. Solid lines are fit of the data by a law of Eq. (4).

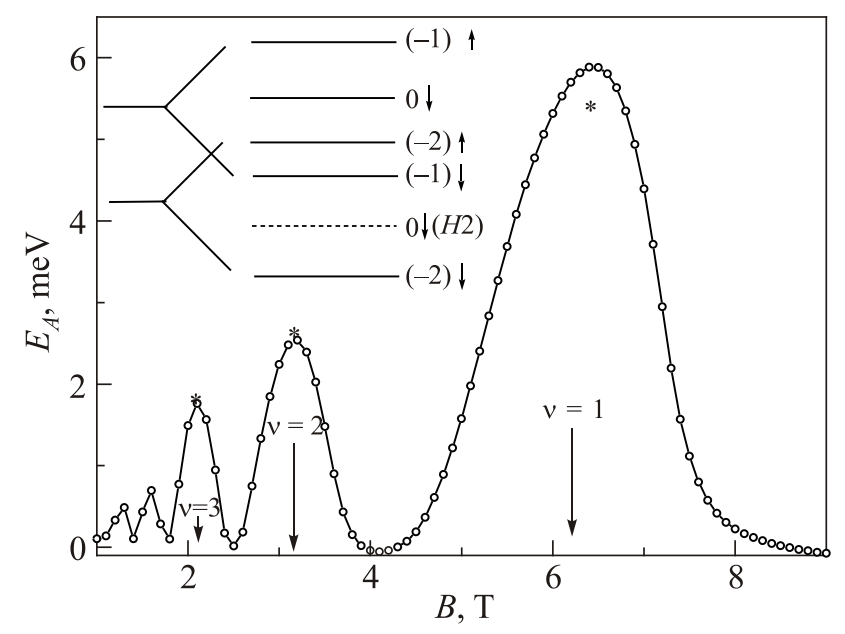

Fig. 4. The magnetic field dependence of experimentally extracted activation energies and theoretically calculated halves of energy gap values for $v=1,2$ and 3 (asterisks). Inset: a scheme of spin level layout for heavy-hole subbands $H 1$ and $H 2$ at investigated structure.

by our k.p model. In particular, we don't observe an explicit manifestation of a strong Rashba spin-orbit splitting caused by an asymmetry of QW confinement potential (see Fig. 1 in [2]).

\subsection{Estimation of parameters}

Let us analyze the obtained results in more details. For $v=1$ we have a gap between $N=0 \downarrow$ LL of $H 2$ subband and $N=-1 \downarrow \mathrm{LL}$ of the $H 1$ subband at $B_{1}=6.3 \mathrm{~T}$ and it is seen geometrically from Fig. 1 that $\Delta_{1}$ should be close in magnitude to the gap between the heavy-hole sizequantized levels $H 1$ and $H 2$ at $B=0$ :

$$
\Delta_{1} \cong E_{H 1}-E_{H 2}=3 \varepsilon_{h},
$$

where $\varepsilon_{h}=\frac{\pi^{2}}{2} \frac{h^{2}}{m_{h h} d^{2}}, m_{h h}$ being the bulk heavy-hole mass. For $m_{h h}=0.25 m_{0}[18]$ and $d=20 \mathrm{~nm}$ we have $3 \varepsilon_{h}=$ $=9.6 \mathrm{meV}$ that is really close to the experimental value of $\Delta_{1} \approx 12 \mathrm{meV}$.

Table 1 . The values of magnetic fields, $B_{v}$, of the experimentally obtained activation energies, $E_{A}$, and corresponding gaps, $\Delta_{V}^{\exp }=2 E_{A}$, of calculated energy gaps, $\Delta_{v}^{\text {teor }}$, and background DOS, $D_{v}$, at LL filling factor $v=1,2$ and 3

\begin{tabular}{c|c|c|c}
\hline \hline Parameters & $v=1$ & $v=2$ & $v=3$ \\
\hline$B_{v}, \mathrm{~T}$ & 6.3 & 3.15 & 2.1 \\
$E_{A}, \mathrm{meV}$ & 6 & 2.4 & 1.5 \\
$\Delta_{v}^{\text {teor }}, \mathrm{meV}$ & 11.6 & 6.9 & 3.8 \\
$\Delta_{v}^{\exp }, \mathrm{meV}$ & 12 & 4.8 & 3 \\
$D_{v}, 10^{10} \mathrm{~cm}^{-2} \cdot \mathrm{meV}^{-1}$ & $0.64 \pm 0.1$ & $0.64 \pm 0.05$ & $0.64 \pm 0.05$ \\
\hline \hline
\end{tabular}


Note that the value of the energy gap at $v=1$, $\Delta_{1} \approx 40 \mathrm{meV}$, extracted in [9] for HgTe QW with $d=11 \mathrm{~nm}$, corresponds well to the estimation by formula (5).

Now we consider the behavior of the mobility gaps between LLs corresponding to filling factors $v=2$ and $v=3$. In accordance with a scheme of LL levels (see inset on Fig. 4) we have:

$$
\Delta_{2}=\Delta_{Z}\left(B_{2}\right)-\Delta_{c}\left(B_{2}\right)
$$

and

$$
\Delta_{3}=2 \Delta_{c}\left(B_{3}\right)-\Delta_{Z}\left(B_{3}\right)
$$

Here $\Delta_{c}$ and $\Delta_{Z}$ are cyclotron and Zeeman splitting, respectively, with $\Delta_{c}=\hbar \omega_{c}, \omega_{c}=e B / m_{c}, m_{c}$ being electron effective mass on the Fermi level, and $\Delta_{Z}=g \mu_{B} B, g$ is Lande factor, $\mu_{B}=e \hbar / 2 m_{0}$ is Bohr magneton; $B_{2}=3.15 \mathrm{~T}$ and $B_{3}=2.1 \mathrm{~T}$. Using the experimental values of $\Delta_{2}$ and $\Delta_{3}$ (see Table 1) and neglecting the $B$-dependence of effective mass and $g$ factor for $B_{3}<B<B_{2}$, we get the following estimates for the parameters: $m_{c} / m_{0}=0.037 \pm 0.005$ and $g=75 \pm 5$.

Note, in a case that Zeeman splitting is under the cyclotron one in the $H 1$ subband (see a scheme of LL levels on the Fig. 1 in Ref. 16) we would have

$$
\Delta_{2}=\Delta_{c}\left(B_{2}\right)-\Delta_{Z}\left(B_{2}\right)
$$

and

$$
\Delta_{3}=\Delta_{Z}\left(B_{3}\right)
$$

Then the following values of the parameters would be received: $m_{c} / m_{0}=0.037$ (the same as in a former case) and $g=28 \pm 2$.

It is known that the theoretical values of parameters at the bottom of a heavy-hole 2D-subband are [22-25]: $g=6 \kappa(\cong 60$ for HgTe $)$ and $m_{0} / m_{c}=\left(\gamma_{1}+\bar{\gamma}\right)\left(m_{c} / m_{0}=\right.$ $=0.047$ for $\mathrm{HgTe})$. But the estimations of the cyclotron and Zeeman energy from the activation analysis in high magnetic fields may strongly contradict these values of $g$ and $m_{c} / m_{0}$ as well as the estimations of the cyclotron energy from the weak field Shubnikov-de Haas $(\mathrm{SdH})$ oscillations due to a mixed nature of heavy-hole subbands at finite $k_{\|}$that leads to a pronounced nonlinearity of the $B$ dependence of the LLs in the inverted-band regime.

The experimentally obtained $g$ and $m_{c} / m_{0}$ values for the inverted-band $\mathrm{HgTe}$ QWs are somewhat contradictory and depend on the density of $2 \mathrm{D}$ electrons and the width of QW.

Thus, for relatively narrow QWs it was found that $g=|20| \pm 5$ for symmetrically doped samples with $d=$ $=9 \mathrm{~nm}\left(n=6.6 \cdot 10^{11} \mathrm{~cm}^{-2}\right)[26]$ and $g \approx 20$ for $11-\mathrm{nm}-$ thick HgTe QW $\left(n=4.66 \cdot 10^{11} \mathrm{~cm}^{-2}\right)$ [9]. For a wide HgTe QW with $d=20 \mathrm{~nm}\left(n=1.5 \cdot 10^{11} \mathrm{~cm}^{-2}\right)$ a value of $|g|=50-60$ is obtained [16].

In [26] the cyclotron mass was determined from the temperature dependence of the $\mathrm{SdH}$ oscillations at low magnetic fields in six $n$-type modulation doped HgTe single quantum wells $(d=4.5 \mathrm{~nm})$. The obtained effective masses are: $0.025 m_{0}$ at $n=(4-6) \cdot 10^{11} \mathrm{~cm}^{-2}$ and $0.034 m_{0}$ at $n=1 \cdot 10^{12} \mathrm{~cm}^{-2}$ in accordance with the theoretical calculations of the semi-classical cyclotron mass based on a $8 \times 8 \mathbf{~ k} \cdot \mathbf{p}$ model.

In [27] the effective mass $m$ has been deduced from the temperature dependence of the amplitudes of the Shubnikov-de Haas (SdH) oscillations to be $m=0.026$ and $0.030 m_{0}$ for $11 \mathrm{~nm}-\mathrm{QW}\left(n=3.45 \cdot 10^{11} \mathrm{~cm}^{-2}\right)$ and $9 \mathrm{~nm}-$ QW $\left(n=6.6 \cdot 10^{11} \mathrm{~cm}^{-2}\right)$, respectively, in good agreement with the values of $m=0.0265$ and $0.0303 m_{0}$, calculated by the authors in the framework of the $8 \times 8 \mathbf{~ k} \cdot \mathbf{p}$ model.

Far-infrared cyclotron resonance photoconductivity (CRP) have been investigated in [28] for HgTe quantum wells of three different widths: 8,16 and $21 \mathrm{~nm}$. Compared to determination of effective masses by the temperature dependence of $\mathrm{SdH}$ oscillations used so far in this material the authors demonstrate that CRP provides a more accurate (about few percents) tool. They found cyclotron resonance effective mass as a function of the density of $2 \mathrm{D}$ electrons: in each sample it increases from the value $(0.0260 \pm$ $\pm 0.0005) m_{0}$ at $n=2.2 \cdot 10^{11} \mathrm{~cm}^{-2}$ to $(0.0335 \pm 0.0005) m_{0}$ at $n=9.6 \cdot 10^{11} \mathrm{~cm}^{-2}$.

\subsection{Density of states in mobility gaps}

In addition to the description of Landau level picture, a direct determination of the density of localized states (DOS) in the mobility gaps is possible from the activation analysis.

The DOS in mobility gaps may be evaluated from the data on activation energy $E_{A}$ as a function of the LL filling factor $v$ [3-6]. As $v=n / n_{B}$ ( $n$ is the electron density, $n_{B}=$ $=e B / h c$ is the degree of LL degeneracy), the filling factor can be tuned by the change of either a carrier density [3] or a magnetic field [4-6].

From the $E_{A}(B)$ dependences the background density of states in the mobility gap may be constructed $[4,6,29]$ :

$$
D_{v}\left(E_{F}\right)=\left[\frac{d E_{A}}{d n}\right]^{-1}=\frac{v e}{2 \pi \hbar c}\left(\frac{d E_{A}(B)}{d B}\right)^{-1} .
$$

Figure 5 shows the results for the DOS at mobility gaps $\Delta_{1}$, $\Delta_{2}$, and $\Delta_{3}$ (see also Table 1). The following features of the mobility gap DOS as a function of energy, $D(E)$, should be mentioned. Even in the middle of a gap when the filling factor is close to an integer, the density of localized states is found to have an unexpectedly high values comparable with the DOS of 2DEG without magnetic field (for a single spin direction):

$$
\begin{gathered}
D_{0}=m / 2 \pi \hbar^{2}=\frac{m}{m_{0}} 1.3 \cdot 10^{26} \mathrm{erg}^{-1} \cdot \mathrm{cm}^{-2}= \\
=\frac{m}{m_{0}} 2.1 \cdot 10^{12} \mathrm{meV}^{-1} \cdot \mathrm{cm}^{-2},
\end{gathered}
$$

which gives $D_{0}=0.63 \cdot 10^{10} \mathrm{meV}^{-1} \cdot \mathrm{cm}^{-2}$ for $m=0.03 m_{0}$. 
Moreover, $D(E)$ remains almost constant in the overwhelming part of the energy intervals between adjacent $\mathrm{LL}: D(E)=(0.58-0.64) \cdot 10^{10} \mathrm{~cm}^{-2} \cdot \mathrm{meV}^{-1} \cong D_{0}$ for $n=1,2$ and 3 (Fig. 5).

The main result that $D(E)$ is practically constant and comparable with $D_{0}$ within the energy interval between adjacent LL's is consistent with the data for structures with n-type conductivity: Si-MOSFET [3], high-mobility [4] and intermediate-mobility [29] AlGaAs/GaAs, InGaAs/InP [5] as well as for multilayer $p-\mathrm{Ge} / \mathrm{Ge}_{1-x} \mathrm{Si}_{x}$ heterostructures [6].

As all short-range impurity potential models lead to an exponential drop in DOS between Landau levels, the clear picture for the DOS in QHE regime may be presented only in terms of the long-range potential fluctuations in combination with the oscillating dependence of DOS on the filling factor. Such idea has been advanced in early work of Shklovskii and Efros [30] and then developed in series of works of Efros with collaborators (see [31,32] and references therein).

In selectively doped heterostructures, the smooth random potential is formed by fluctuations in concentration of remote impurities. When the filling factor is close to an integer very small concentration of electrons, $n<<n_{B}$, can be redistributed in space and thus one occurs in conditions of so called nonlinear screening [30-32] ("threshold" screening in terms of [33]).

For integer $v$ exactly the screening is realized only due to electrons (and holes) induced by an overlap of adjacent fluctuating Landau levels, and so the amplitude of random potential is of the order of corresponding LL gap. Consequently the apparent (averaged over the sample) background DOS becomes of the order of $D_{0}$.

It is considered that for the filling factors close to half integers (the regions of plateau to plateau transition) the potential fluctuations would be small due to effective (linear) electron screening [30-33].

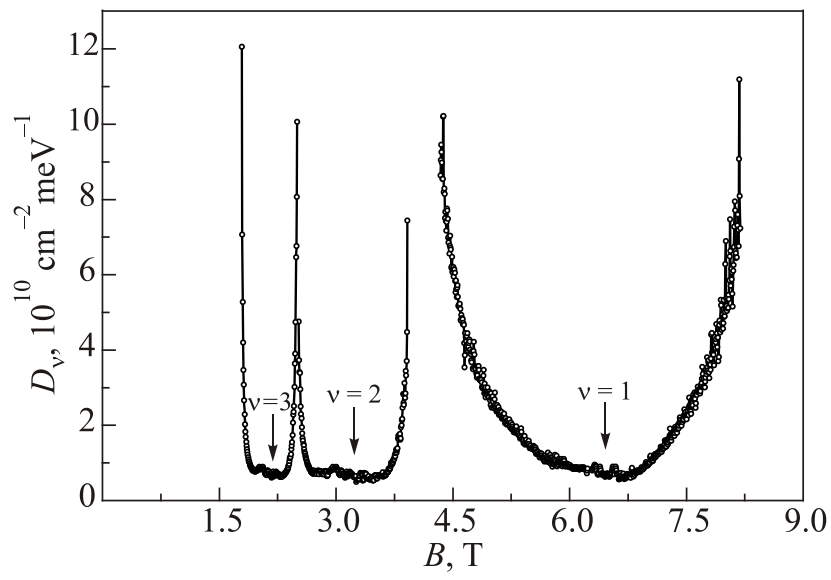

Fig. 5. The background density of states $D_{v}$ for the investigated sample, as deduced from the activation energy dependence on $B$, the integer $v=1,2$ and 3 corresponds to the middle of an energy interval between two LLs.

\section{Conclusions}

Reach information concerning QWs in the 2D heterosystem may be obtained from the study of magnetotransport in the quantum Hall regime. In comparison with a usual analysis of the experimental magnetoresistivity traces the activation analysis yields an accurate quantitative tool to probe inter-LL distances and to estimate the background density of states in mobility gap between adjacent LLs.

We have investigated the activated magnetotransport in the QHE plateau regions at the $\mathrm{HgCdTe} / \mathrm{HgTe} / \mathrm{HgCdTe}$ system with the inverted band structure in magnetic fields up to $9 \mathrm{~T}$ for a wide temperature range $T=2.9-50 \mathrm{~K}$, which turned out possible owing to the high-energy scale of the quantum Hall effect in $\mathrm{HgTe}$.

The activation energies of $\rho_{x x}$ in the QHE regime for the gap between $N=0 \downarrow$ LL of heavy-hole subband $H 2$ and $N=-1 \downarrow$ LL of heavy-hole subband $H 1(v=1)$ and for the gaps $N=-1 \downarrow \rightarrow N=-2 \uparrow(\vee=2)$ and $N=-2 \uparrow \rightarrow N=0 \downarrow$ $(v=3)$ of the $H 1$ subband were determined. It is shown that the experimentally obtained energy gaps are well described within the framework of $\mathbf{k} \cdot \mathbf{p}$ model.

Activated behavior in the quantum Hall regime also yields the background DOS that characterizes random potential distribution in the system.

Note that the temperature ranges where the QHE is still observed are significantly different for various materials: from liquid- and subliquid-helium temperatures for III-V structures to room temperatures for single-layer graphene (see, e.g., [34]). For the studied structure based on HgTe, the QHE range extends up to nitrogen temperatures (see, e.g., [8]) due to large cyclotron and Zeeman splittings.

The research was carried out within the state assignment of FASO of Russia (theme "Spin" No. 01201463330), supported in part by RFBR (project No. 17-02-00330). Samples were grown on the funds of the project "Fundamental Research of Ural Branch of Russian Academy of Sciences, No 15-9-2-21".

1. M. Konig, S. Wiedmann, C. Brne, A. Roth, H. Buhmann, L.W. Molenkamp, X.-L. Qi, and S.-C. Zhang, Science 318, 766 (2007).

2. E.G. Novik, A. Pfeuffer-Jeschke, T. Jungwirth, V. Latussek, C.R. Becker, G. Landwehr, H. Buhmann, and L.W. Molenkamp, Phys. Rev. B 72, 035321 (2005).

3. M.G. Gavrilov and T.V. Kukushkin, Pis'ma JETF 43, 79 (1986).

4. D. Weiss, E. Stahl, G. Weiman, K. Ploog, and K. von Klitziug, Surf. Sci. 170, 285 (1986).

5. H.P. Wei, A.M. Chang, D.C. Tsui, and M. Rozeghi, Phys. Rev. B 32, 7016 (1985).

6. Yu.G. Arapov, G.I. Harus, V.N. Neverov, N.G. Shelushinina, M.V. Yakunin, G.A. Alshanskii, and O.A. Kuznetsov, Nanotechnology 11, 351 (2000). 
7. Yu.G. Arapov, G.I. Harus, V.N. Neverov, N.G. Shelushinina, M.V. Yakunin, and O.A. Kuznetsov, JETP 96, 118 (2003).

8. D.A. Kozlov, Z.D. Kvon, N.N. Mikhailov, S.A. Dvoretskii, S. Weishäupl, Y. Krupko, and J.-C. Portal, Appl. Phys. Lett. 105, 132102 (2014).

9. T. Khouri, M. Bendias, P. Leubner, C. Brüne, H. Buhmann, L.W. Molenkamp, U. Zeitler, N.E. Hussey, and S. Wiedmann, Phys. Rev. B 93, 125308 (2016).

10. Y.G. Arapov, S.V. Gudina, V.N. Neverov, S.M. Podgornykh, M.R. Popov, G.I. Harus, N.G. Shelushinina, M.V. Yakunin, N.N. Mikhailov, and S.A. Dvoretsky, Semiconductors 49, 1593 (2015).

11. Yu.G. Arapov, S.V. Gudina, V.N. Neverov, S.M. Podgornykh, M.R. Popov, G.I. Harus, N.G. Shelushinina, M.V. Yakunin, S.A. Dvoretsky, and N.N. Mikhailov, J. Low Temp. Phys. 185, 665(2016).

12. M. Konig, H. Buhmann, L. Molenkamp, and T. Hughes, $J$. Phys. Soc. Jpn. 77, 031007 (2008).

13. M.I.D'yakonov and A.V. Khaetskii, JETP 55, 917 (1982).

14. L.G. Gerchikov and A. Subashiev, Phys. Status Solidi B 160, 443 (1990).

15. E.B. Olshanetsky, S. Sassine, Z.D. Kvon, N.N. Mikhailov, S.A. Dvoretsky, J.C. Portal, and A.L. Aseev, Pis'ma v JETF 84, 661 (2006); Z.D. Kvon, E.B. Olshanetsky, N.N. Mikhailov, and D.A. Kozlov, Fiz. Nizk. Temp. 35, 10 (2009) [Low Temp. Phys. 35, 6 (2009)].

16. M.V. Yakunin, S.M. Podgornykh, N.N. Mikhailov, and S.A. Dvoretsky, Physica E 42, 948 (2010).

17. Yu.G. Arapov, N.A. Gorodilov, V.N. Neverov, M.V. Yakunin, A.V. Germanenko, G.M. Min'kov, O.A. Kuznetsov, R.A. Rubtsova, A.L. Chernov, and L.K. Orlov, JETP Lett. 59, 268 (1994).

18. Y. Guldner, C. Rigaux, M. Grynberg, and A. Mycielski, Phys. Rev. B 8, 3875 (1973).
19. F. Ancilotto, A. Fasolino, and J.C. Maan, Phys. Rev. B 38, 1788(1988).

20. M. Schultz, U. Merkt, A. Sonntag, U. Rössler, R. Winkler, T. Colin, P. Helgesen, T. Skauli, and S. Løvold, Phys. Rev. B 57, 14772 (1998).

21. J.C. Hensel and K. Suzuki, Phys. Rev. B 9, 4219 (1974).

22. R.W. Martin, R.J. Nicholas, G.J. Rees, S.K. Heywood, N.J. Mason, and P.J. Walker, Phys. Rev. B 42, 9237 (1990); R.J. Warburton, R.W. Martin, R.J. Nicholas, L.K. Howard, and M.T. Emeny, Semicond. Sci. Technol. 6, 359 (1991).

23. N.A. Gorodilov, O.A. Kuznetsov, L.K. Orlov, R.A. Rubtsova, A.L. Chernov, N.G. Shelushinina, and G.L. Shtrapenin, JETP Lett. 56, 394 (1992).

24. M.A. Semina and R.A. Suris, Semiconductors 49, 797 (2015).

25. T. Wimbauer, K. Oettinger, A.L. Efros, B.K. Meyer, and H. Brugger, Phys. Rev. B 50, 8889 (1994).

26. A. Pfeuffer-Jeschke, F. Goschenhofer, S.J. Cheng, V. Latussek, J. Gerschütz, C.R. Becker, R.R. Gerhardts, and G. Landwehr, Physica B 256-258, 486 (1998).

27. X.C. Zhang, A. Pfeuffer-Jeschke, K. Ortner, V. Hock, H. Buhmann, C.R. Becker, and G. Landwehr, Phys. Rev. B 63, 245305 (2001).

28. Z.-D. Kvon, S.N. Danilov, N.N. Mikhailov, S. Dvoretsky, W. Prettl, and S. Ganichev, cond-mat/0708.2175.

29. P. Svoboda, G. Nachtwei, C. Breitlow, S. Heide, and M. Cukr, cond-mat/9612053.

30. B.I. Shklovskii and A.L. Efros, JETP Lett. 44, 669 (1986).

31. A.L. Efros, Solid State Commun. 70, 253 (1989).

32. A.L. Efros, F.G. Pikus, and V.G. Burnett, Phys. Rev. B 47, 2233 (1993).

33. V. Kukushkin, S.V. Meshkov, and V.B. Timofeev, Phys. Usp. 31, 511 (1988).

34. K.S. Novoselov, A.K. Geim, S.V. Morozov, D. Jiang, M.I. Katsnelson, I.V. Grigorieva, S.V. Dubonos, and A.A. Firsov, Nature 438, 197 (2005). 